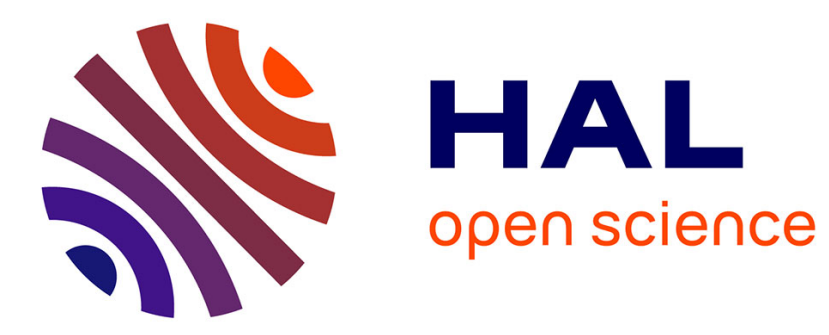

\title{
Modeling Sleep Mode Gains in Energy-Aware Networks
}

Luca Chiaraviglio, Delia Ciullo, Marco Mellia, Michela Meo

\section{To cite this version:}

Luca Chiaraviglio, Delia Ciullo, Marco Mellia, Michela Meo. Modeling Sleep Mode Gains in EnergyAware Networks. [Research Report] RR-8187, INRIA. 2012. hal-00759403

\section{HAL Id: hal-00759403 \\ https://hal.inria.fr/hal-00759403}

Submitted on 17 Dec 2012

HAL is a multi-disciplinary open access archive for the deposit and dissemination of scientific research documents, whether they are published or not. The documents may come from teaching and research institutions in France or abroad, or from public or private research centers.
L'archive ouverte pluridisciplinaire HAL, est destinée au dépôt et à la diffusion de documents scientifiques de niveau recherche, publiés ou non, émanant des établissements d'enseignement et de recherche français ou étrangers, des laboratoires publics ou privés. 


\section{Modeling Sleep Mode} Gains in Energy-Aware Networks

Luca Chiaraviglio, Delia Ciullo, Marco Mellia, Michela Meo

\section{RESEARCH}





\title{
Inĩia
}

\section{Modeling Sleep Mode Gains in Energy-Aware Networks}

\author{
Luca Chiaraviglio*, Delia Ciullo ${ }^{\dagger}$, Marco Mellia $^{\ddagger}$, Michela Meo ${ }^{\S}$
}

Project-Teams Maestro

Research Report $\mathrm{n}^{\circ} 8187$ - December 2012 - 28 pages

A preliminary version of this paper has appeared at IEEE INFOCOM 2011 Workshop on Green Communications and Networking [9].

* Inria Sophia Antipolis, France, luca.chiaraviglio@inria.fr

† Inria Sophia Antipolis, France, delia.ciullo@inria.fr

¥ Politecnico di Torino, Italy, mellia@tlc.polito.it

$\S$ Politecnico di Torino, Italy, michela.meo@polito.it

\section{RESEARCH CENTRE}

SOPHIA ANTIPOLIS - MÉDITERRANÉE

2004 route des Lucioles - BP 93

06902 Sophia Antipolis Cedex 


\begin{abstract}
Nowadays two main approaches are being pursued to reduce energy consumption of networks: the use of sleep modes in which devices enter a low-power state during inactivity periods, and the adoption of energy proportional mechanisms where the device architecture is designed to make energy consumption proportional to the actual load. Common to all the proposals is the evaluation of energy saving performance by means of simulation or experimental evidence, which typically consider a limited set of benchmarking scenarios.

In this paper, we do not focus on a particular algorithm or procedure to offer energy saving capabilities in networks, but rather we formulate a theoretical model based on random graph theory that allows to estimate the potential gains achievable by adopting sleep modes in networks where energy proportional devices are deployed. Intuitively, when some devices enter sleep modes some energy is saved. However, this saving could vanish because of the additional load (and power consumption) induced onto the active devices. The impact of this effect changes based on the degree of load proportionality. As such, it is not simple to foresee which are the scenarios that make sleep mode or energy proportionality more convenient.

Instead of conducting detailed simulations, we consider simple models of networks in which devices (i.e., nodes and links) consume energy proportionally to the handled traffic, and in which a given fraction of nodes are put into sleep mode. Our model allows to predict how much energy can be saved in different scenarios. The results show that sleep modes can be successfully combined with load proportional solutions. However, if the static power consumption component is one order of magnitude less than the load proportional component, then sleep modes become not convenient anymore. Thanks to random graph theory, our model gauges the impact of different properties of the network topology. For instance, highly connected networks tend to make the use of sleep modes more convenient.
\end{abstract}

Key-words: Energy-efficient networks, sleep modes, random graph models 


\section{Modélisation des gains possibles avec les modes de sommeil dans les réseaux énergétiquement performants}

Résumé : Pour réduire la consommation d'énergie des réseaux, il existe deux approches principales: l'utilisation d'un mode veille à basse consommation énergétique pendant les périodes d'inactivité et l'adoption de matériels dont la consommation d'énergie est proportionnelle à la charge. Dans ce rapport, nous formulons un modèle théorique, basé sur la théorie des graphes aléatoires, qui permet d'estimer les gains potentiels réalisables en adoptant des modes de mise en veille dans les réseaux où sont déployés des appareils consommant de l'énergie proportionnellement à leur charge. La mise en veille de certains appareils permet d'économiser sur l'énergie de ceux-ci, mais en contrepartie l'énergie consommée par les appareils actifs augmentera au vu de leur charge accrue. Le degré de proportionnalité entre la charge d'un appareil et sa consommation d'énergie joue un rôle important. Ainsi, il n'est pas simple de prévoir les cas dans lesquels une mise en veille ou une consommation proportionnelle à la charge serait plus avantageuse. Notre approche se base non pas sur des simulations détaillées mais sur des modèles simples de réseaux dans lesquels le matériel (nœuds et liens) consomme de l'énergie proportionnellement au trafic géré et dans lesquels une certaine fraction de nœuds est mise en veille. Notre modèle permet d'estimer l'économie d'énergie dans des scénarios divers. Nos résultats montrent que la mise en veille et la proportionnalité de la consommation avec la charge peuvent être combinées avec succès. Toutefois, si la puissance de consommation statique est un ordre de grandeur inférieure à celle proportionnelle à la charge, la mise en veille perd ses avantages. Grâce à la théorie des graphes aléatoires, l'impact de différentes propriétés de la topologie du réseau est étudié. A titre d'exemple, un réseau fortement connexe avantage la mise en veille.

Mots-clés : réseaux énergétiquement performants, modes de sommeil, modèles des graphes aléatoires 


\section{Introduction}

In networking, one of the main causes of energy waste is the fact that most of the devices do not consume energy proportionally to the work they sustain, but they consume much even when they are under-utilized [1]. On the contrary, network usage and traffic follow the typical human being activity patterns, with significant differences between peak and off-peak values and typical daily periodicities. The network results thus highly under-utilized for long periods of time causing a large energy waste. Many solutions are being studied to reduce this waste, or, equivalently, to make the network consumption proportional to the traffic load [2]. The proposed approaches can be divided into two main categories: i) Energy proportional approaches work on the individual devices and try to achieve energy consumption proportionality by adapting the speed (and capacity) of the devices to the actual load, over relatively short time-scales [3]; ii) Sleep mode approaches involve the network as a whole and approximate load proportionality by carefully distributing the traffic in the network so that some devices are highly utilized while others become idle and are put in sleep modes [4]. The two solutions can be merged so that energy proportional devices are present and sleep mode can be leveraged to possibly save additional energy.

In this context, several solutions have been proposed in the literature, including network management algorithms that optimize traffic routing so as to maximize the energy saving offered by sleep mode enabled devices (see Sec. 7 for more details). However, to the best of our knowledge, all previous works adopt simulation or actual testbed experiments as main means to assess energy saving performance, and typically few benchmarking scenarios have been considered. Furthermore, either energy proportionality or sleep modes approach is assumed, with few works only considering the combination of the two. In this paper, we instead aim at: i) comparing and combining the two approaches, and ii) proposing an analytical methodology to estimate their benefits.

Given a family of network topologies, and given a model of the energy consumed by a device as a function of its load, is it better to purely rely on device energy proportionality capability, or, on the contrary, is it better to couple it with sleep mode solutions? And, also, which is the minimum energy proportionality that would make sleep mode ineffective? What is the impact of the network size, or topological properties on the benefits of energy saving feature? The answer to these questions is the goal of this paper.

When a device is switched-off the traffic passing through it has to be rerouted on different, typically longer, paths; thus, the beneficial saving achieved by switching off the device is mitigated by the increase of the consumption of the devices that remain on, due to the higher load they have to sustain. To investigate this trade-off, we consider a general model to represent network topologies ranging from backbone networks to metropolitan networks, and a general model for device power consumption.

Several power consumption models for devices have been proposed [5], [6], [7], [8]. Basically, all these models assume that the energy consumption of network devices, i.e., nodes and links, is composed by a constant amount and a variable part that is an increasing function of the traffic that flows through the devices. We compute the total network energy cost as the sum of the fixed and variable cost of network devices which depends on the traffic they have to carry. In our previous work [9] this problem has been faced considering that only links offer energy saving capabilities. In this work we go a step further by considering a more general model that includes also the cost of nodes. We start considering the variable cost of devices scales linearly with the load. Then, we extend the model to generic cost functions which include linear and super-linear costs. The network and its topological characteristics are represented by random graphs; leveraging then on random graph theory, the load on network devices is computed from 
the knowledge of the shortest path between node pairs. Thus, the energy consumption of the whole network is easily derived.

Since we are not interested in proposing a novel algorithm to select which link and node can be put into sleep mode, we consider a generic policy, according to which some elements are turned off. This results in a change in the topological characteristics of the network, which is modeled as a new graph, whose energy consumption is evaluated using graph theory again. To the best of our knowledge, the only previous work that is similar to ours is [8]. Yet, only simple simulations have been used, so that the set and generality of presented results is limited. In our work, we present modeling results that corroborate the intuition of [8] and derive more general insights.

We present an extensive sensitivity analysis to show the impact of model parameters. We include both small-world and power-law graph models that are claimed to reflect actual network topology properties [10]. Our results show that:

- when the variable part of the cost model is small with respect to the constant part, as is typical of today devices, sleep modes are convenient;

- for future devices, whose consumption will probably be more load proportional, sleep modes might not be convenient anymore provided the static cost would be one order of magnitude smaller than the variable part;

- network topology characteristics have limited impact on energy saving. Yet, well-connected topologies show larger benefits in terms of energy saving margins when sleep-mode policies are in place.

This suggests that, given the today technological constraints that make the constant energy consumption of devices quite large, sleep mode enabled networks will allow to save more energy than purely energy proportional approaches for long time. Finally, we emphasize that, despite being simple, our model gives general insights of sleep modes effectiveness in actual telecommunication networks.

The rest of the paper is organized as follows. The system model and methodology is detailed in Section 2. The adopted network models are reported in Section 3. We present the evaluation of sleep modes in Section 4. The comparison of different switching off policies is reported in Section 5. A discussion about model assumptions is reported in Section 6. Section 7 reviews related work. Finally, Section 8 concludes the paper.

\section{System Model and Methodology}

In this section we provide a general overview of the methodology we use to evaluate sleep mode gains.

The network is composed by access and transport devices. Access devices are the possible sources and destinations of traffic, therefore they can never be powered off. On the contrary, some of transport devices can be turned off if their traffic can be supported by other devices that remain on.

We adopt the following assumptions: i) traffic is uniformly exchanged among all access nodes; ii) traffic is routed on the shortest paths among nodes ${ }^{1}$; iii) the set of devices to be switched off is given a-priori, e.g., it has been previously chosen based on collection of traffic measurements and predictions so as to guaranteeing the minimum required Quality of Service (QoS); iv) node/link power consumption is composed by a fixed amount of power, and a variable part that is an

\footnotetext{
${ }^{1}$ An energy-aware routing protocol could be adopted here rather than a shortest path routing. For example, traffic could be routed over the paths consuming the lowest power. However, this issue is outside the scope of this paper.
}

$\mathrm{RR} \mathrm{n}^{\circ} 8187$ 
increasing function of the current traffic flowing on the link; v) the same power consumption model is applied to all the links/nodes in the network.

Modeling the power consumption of today's networks is still an open issue, since it mainly depends on the technology of network devices. Current network devices consume a large amount of static power and a limited amount of power that depends on the current load [1]. However, future devices will instead be more energy-proportional [2], with a predominant term of power scaling with load. To capture the effectiveness of sleep modes in both the aforementioned scenarios, we first assume a linear power consumption model, in accordance with [8]. Then, as a second step, we extend our model to super-linear power functions, focusing on the case in which power scales quadratically with load. The latter is representative of devices exploiting Dynamic Voltage Scaling (DVS) techniques [7]. This allows us to investigate the impact of sleep modes when extremely optimized devices are deployed.

\subsection{Basic formulation and metrics}

Let the transport network topology be described by an undirected graph $G(\mathcal{N}, \mathcal{L})$, with $\mathcal{N}$ the set of nodes, with cardinality $N=|\mathcal{N}|$, and $\mathcal{L}$ the set of links, with cardinality $L=|\mathcal{L}|$. The average node degree is $\bar{K}=\frac{2 L}{N}$. The link rate is denoted by $R$, while $T$ is the total traffic ${ }^{2}$ flowing in the transport network from access nodes at a given time.

The $j-t h$ link power consumption, $A(j)$, is modeled by a fixed amount $F$ and a variable term that is a function $f(\cdot)$ of the link load, $\rho(j)$ :

$$
A(j)=F+f(\rho(j))
$$

For the sake of simplicity, we assume the same fixed power $F$ and function of the load $f(\cdot)$ for all links. Similarly, the $i-t h$ node power consumption $A_{N}(i)$ is modeled by a fixed amount $F_{N}$ and a variable term that is a function $g(\cdot)$ of the node load $\rho_{N}(i)$ :

$$
A_{N}(i)=F_{N}+g\left(\rho_{N}(i)\right)
$$

Again, the same fixed power $F_{N}$ and function $g(\cdot)$ is assumed for all the nodes ${ }^{3}$. The total network consumption, $C$, can be computed as

$$
\begin{aligned}
& C=\sum_{j \in \mathcal{L}}(F+f(\rho(j)))+\sum_{i \in \mathcal{N}}\left(F_{N}+g\left(\rho_{N}(i)\right)\right)= \\
& L F+\sum_{j \in \mathcal{L}} f(\rho(j))+N F_{N}+\sum_{i \in \mathcal{N}} g\left(\rho_{N}(i)\right)
\end{aligned}
$$

Let us consider the case where the variable part of the cost model increases linearly with the load. For links we have:

$$
f(\rho(j))=\alpha \rho(j)
$$

thus,

$$
\sum_{j \in \mathcal{L}} f(\rho(j))=\sum_{j \in \mathcal{L}} \alpha \rho(j)=L \alpha E[\rho]=L \alpha \bar{\rho}
$$

\footnotetext{
${ }^{2}$ We assume values for $R$ and $T$ such that the network is not overloaded, i.e., the link load $\rho$ is smaller than one.

${ }^{3}$ Note that this model can be easily extended to integrate different classes of links (nodes), each of them with a different value of the parameters $F\left(F_{N}\right)$.
} 


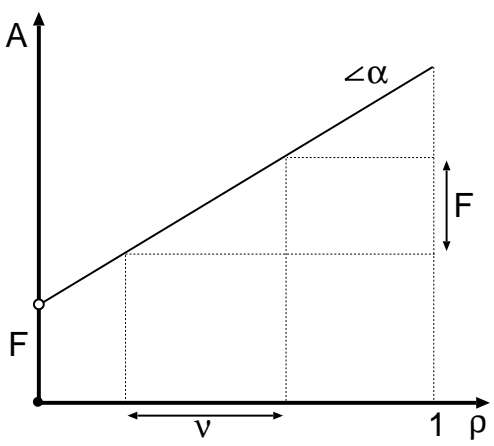

Figure 1: Linear power consumption model for a link.

where $\bar{\rho}$ is the mean link load and $\alpha$ is a scaling coefficient. Similarly, for nodes we assume:

$$
g\left(\rho_{N}(i)\right)=\beta \rho_{N}(i)
$$

By assuming that the node variable power consumption depends on the total amount of traffic passing through the node and that the link load is independent on the node degree, we define the average load on a node $\bar{\rho}_{N}$ as the sum of the average load carried by the $\bar{K}$ links connected on average to a node: $\bar{\rho}_{N}=\bar{K} \bar{\rho}$.

Under these assumptions, the total network consumption is

$$
C=N \frac{\bar{K}}{2}(F+\alpha \bar{\rho})+N\left(F_{N}+\beta \bar{K} \bar{\rho}\right)
$$

As an index of the relative importance of variable and fixed costs, we define the constant cost equivalent load,

$$
\nu=\frac{F}{\alpha} ; \quad \nu_{N}=\frac{F_{N}}{\beta}
$$

where $\nu\left(\nu_{N}\right)$ is the amount of load that, added to a link (node), makes its energy consumption increase of a quantity $F\left(F_{N}\right)$. Or, in other terms, whenever the load increases by an amount $\nu\left(\nu_{N}\right)$, the part of the energy consumption due to links (nodes) increases by $F\left(F_{N}\right)$. The parameters $\nu, \nu_{N}$ and their ratio play a crucial role in the evaluation of sleep mode schemes, as we show in Section 4. Fig. 1 shows a graphical representation of a link power consumption $A$.

The average link load can be computed as:

$$
\bar{\rho}=\frac{T \bar{d}}{L R}
$$

where $\bar{d}$ is the average shortest path length and $L R$ is the total capacity offered by the network. We call $C$ the all-on network consumption and we take this value as a reference for the nominal consumption of the network. Table 1 summarizes the notation introduced so far.

\subsection{Node Switch-off Policy}

We now compute the network consumption when some nodes enter sleep mode. We assume that the scheme according to which the nodes enter sleep mode has been preliminarily planned so that, when the nodes are removed (powered off), the resulting network still meets the QoS 


\begin{tabular}{|c|c|}
\multicolumn{2}{c}{ Table 1: Notation } \\
\hline Symbol & Definition \\
$L$ & number of nodes \\
$\bar{K}$ & number of links \\
$T$ & average node degree \\
$R$ & traffic \\
$F\left(F_{N}\right)$ & link rate \\
$A\left(A_{N}\right)$ & link (node) fixed cost \\
$\bar{\rho}\left(\bar{\rho}_{N}\right)$ & link (node) total cost \\
$\bar{d}$ & average link (node) load \\
$\alpha(\beta)$ & link (node) scaling coefficient \\
$C$ & network consumption \\
$\nu\left(\nu_{N}\right)$ & link (node) constant cost equivalent load \\
\hline
\end{tabular}

constraints, e.g., the graph is connected and maximum link load is below the admissible value. Clearly, when a node is powered off, all links connected to it are switched off too.

Let $p \in(0,1)$ be the fraction of nodes that are switched off. We model the network resulting from the sleep mode scheme through the new graph in which we randomly remove a fraction $p$ of the nodes; we take $p$ smaller than the critical probability after which the network becomes disconnected. In this regime, random node elimination makes the new graph maintain the same structure of the original graph; refer to [11] for details. The number of nodes after the random node removal becomes $N^{\prime}=N(1-p)$, and the new average degree becomes $\bar{K}^{\prime}=\bar{K}(1-p)$. From (7), the average network consumption in sleep mode $C^{\prime}$ is now:

$$
\begin{aligned}
& C^{\prime}=N \frac{\bar{K}}{2}(1-p)^{2}\left(F+\alpha \bar{\rho}^{\prime}\right)+ \\
& N(1-p)\left(F_{N}+\beta \bar{K}(1-p) \bar{\rho}^{\prime}\right)
\end{aligned}
$$

with

$$
\bar{\rho}^{\prime}=T \frac{\bar{d}^{\prime}}{N^{\prime} \frac{\bar{K}^{\prime}}{2} R}=T \frac{\bar{d}^{\prime}}{N \frac{\bar{K}}{2}(1-p)^{2} R}
$$

where $\bar{d}^{\prime}$ is the average shortest path length in the new graph.

Our aim now is to compare the energy consumption of the all-on network consumption, $C$, to the one of the network in sleep modes, $C^{\prime}$. To this purpose, we define the ratio $E=C^{\prime} / C$ as the energy reduction ratio. Intuitively, the use of sleep modes for network devices saves energy when $E<1$.

By computing $E$ and comparing $C$ and $C^{\prime}$ in (7) and (10), it is possible to evaluate when sleep modes are convenient:

$$
\begin{aligned}
& C>C^{\prime} \quad \text { iff } \quad N \frac{\bar{K}}{2}(F+\alpha \bar{\rho})+N\left(F_{N}+\beta \bar{K} \bar{\rho}\right)> \\
& N \frac{\bar{K}}{2}(1-p)^{2}\left(F+\alpha \bar{\rho}^{\prime}\right)+N(1-p)\left(F_{N}+\beta \bar{K}(1-p) \bar{\rho}^{\prime}\right)
\end{aligned}
$$

Then, we have

$$
C>C^{\prime} \quad \text { iff } \quad \frac{F+\frac{2}{K(2-p)} F_{N}}{\alpha+2 \beta}>\frac{T}{\left(2 p-p^{2}\right) N \frac{K}{2} R}\left(\bar{d}^{\prime}-\bar{d}\right)
$$

This equation defines the region in which the sleep mode approach is convenient. 
Note that, if the network technology is such that the link cost is negligible with respect to the node cost, i.e., $F<<F_{N}$ and $\alpha<<\beta$, we can disregard the link cost and we have

$$
C>C^{\prime} \quad \text { iff } \quad \nu_{N}>\frac{2 T}{p N R}\left(\bar{d}^{\prime}-\bar{d}\right)
$$

Similarly, if the node cost is negligible with respect to the link cost, i.e., $F_{N}<<F$ and $\beta<<\alpha$, we obtain

$$
C>C^{\prime} \quad \text { iff } \quad \nu>\frac{T}{\left(2 p-p^{2}\right) N \frac{\bar{K}}{2} R}\left(\bar{d}^{\prime}-\bar{d}\right)
$$

Lemma 1: If devices do not implement load proportionality $(\alpha=\beta=0)$, sleep mode is always convenient, i.e., $C^{\prime}<C$ and $E<1$.

Proof. With $\alpha=\beta=0$, (12) simplifies to $N \frac{\bar{K}}{2} F+N F_{N}>N \frac{\bar{K}}{2}(1-p)^{2} F+N(1-p) F_{N}$, which is verified for any $p \in(0,1)$.

Lemma 2: If the fixed power consumption is zero $\left(F=F_{N}=0\right)$, sleep mode is never convenient, i.e., $E \geq 1$.

Proof. If $F=F_{N}=0,(12)$ becomes: $\bar{d}>\bar{d}^{\prime}$, i.e., it is verified if the average shortest path after some node switch off, $\bar{d}^{\prime}$ is smaller than the initial average shortest path $\bar{d}$, which is never true ${ }^{4}$. Consequently, $E \geq 1$.

\subsection{Generalization of the cost function}

We now extend our analysis to a generic cost model, in which the variable part of the cost increases according to a polynomial function of the load. For simplicity, we first consider the cost due to links and then compute the total cost due to nodes too. For each link $j$, we can write the cost function as:

$$
A(j)=F+\sum_{z=1}^{\infty} \alpha_{z}(\rho(j))^{z}
$$

The total cost $C$ becomes:

$$
\begin{aligned}
& C=\sum_{j \in \mathcal{L}}\left(F+\sum_{z=1}^{\infty} \alpha_{z}(\rho(j))^{z}\right)+\sum_{i \in \mathcal{N}}\left(F_{N}+\sum_{z=1}^{\infty} \beta_{z}\left(\rho_{N}(i)\right)^{z}\right)= \\
& L F+\sum_{j \in \mathcal{L}} \sum_{z=1}^{\infty} \alpha_{z}(\rho(j))^{z}+N F_{N}+\sum_{i \in \mathcal{N}} \sum_{z=1}^{\infty} \beta_{z}\left(\rho_{N}(i)\right)^{z}= \\
& L F+L \sum_{z=1}^{\infty} \alpha_{z} E\left[\rho^{z}\right]+N F_{N}+N \sum_{z=1}^{\infty} \beta_{z} E\left[\rho_{N}^{z}\right]
\end{aligned}
$$

where the last term $E\left[\rho_{N}^{z}\right]$ can be computed as:

$$
\begin{aligned}
& E\left[\rho_{N}^{z}\right]=\sum_{k=1}^{\infty} E\left[\rho_{N}^{z} \mid K=k\right] \mathbb{P}(K=k)= \\
& \sum_{k=1}^{\infty} E\left[\left(\sum_{q=1}^{k} \rho(q)\right)^{z}\right] \mathbb{P}(K=k),
\end{aligned}
$$

\footnotetext{
${ }^{4}$ Note that, in our context, $\bar{d}^{\prime} \geq \bar{d}$ is always verified since we are considering only connected graphs, and we assume we cannot remove source/destination nodes.
} 
where $K$ is the random variable representing the degree distribution. The last result follows from the node load computation: we assume that the load of a node is given by the sum of the load carried by the links connected to it.

Observe that, given all the moments of the link load distribution and the degree distribution up to the $z$-th moment, (17) allows the computation of the total cost for any type of polynomial cost function. Moreover, we can extend the previous analysis to any other cost functions, e.g., logarithmic or any sub-linear functions, by using the Maclaurin series to approximate the cost function, so that cost can still be expressed by (16).

Quadratic Cost Model As a special case of (16), we assume that the variable part of the cost model increases quadratically with the load. Thus, the $j-t h$ link cost can be modeled as: $F+\alpha(\rho(j))^{2}$ and (3) becomes:

$$
\begin{aligned}
& C=L F+\alpha \sum_{j \in \mathcal{L}}(\rho(j))^{2}+N F_{N}+\beta \sum_{i \in \mathcal{N}}\left(\rho_{N}(i)\right)^{2}= \\
& L\left(F+\alpha E\left[\rho^{2}\right]\right)+N\left(F_{N}+\beta E\left[\rho_{N}^{2}\right]\right)
\end{aligned}
$$

Considering the node load distribution, we have: $E\left[\rho_{N}^{2}\right]=\sigma_{\rho_{N}}^{2}+E\left[\rho_{N}\right]^{2}=\sigma_{\rho_{N}}^{2}+(\bar{\rho} \bar{K})^{2}$, where $\sigma_{\rho_{N}}^{2}$ is the variance of the node load distribution. Note that, being the node load given by the sum of the load of the node's links, the node load distribution is a compound distribution that depends on the link load and the degree distributions. Assuming that link load $\rho$ is independent on the node degree $K$, and that the load on the links are i.i.d. random variables, we obtain: $\sigma_{\rho_{N}}^{2}=\bar{K} \sigma_{\rho}^{2}+\bar{\rho}^{2} \sigma_{K}^{2}$. Thus,

$$
E\left[\rho_{N}^{2}\right]=(\bar{\rho} \bar{K})^{2}+\bar{K} \sigma_{\rho}^{2}+\bar{\rho}^{2} \sigma_{K}^{2}=\bar{\rho}^{2} E\left[K^{2}\right]+\sigma_{\rho}^{2} \bar{K}
$$

Considering the link load distribution we have: $E\left[\rho^{2}\right]=E[\rho]^{2}+\sigma_{\rho}^{2}=\bar{\rho}^{2}+\sigma_{\rho}^{2}$, where $\sigma_{\rho}^{2}$ is the variance of the link load distribution. From (9), we can observe that $\bar{\rho}$ is a linear function of the average shortest path $\bar{d}$. Thus, $\bar{\rho}=E[\rho]=E[(T / L R) d]=(T / L R) E[d]$, and the link load variance is:

$$
\sigma_{\rho}^{2}=\left(\frac{T}{L R}\right)^{2} \sigma_{d}^{2},
$$

where $\sigma_{d}^{2}$ is the variance of the shortest path distribution.

Therefore, by knowing the first and the second moments of the shortest path and the degree distribution, we can compute the total cost given by (19). Note that to compute the network cost after the random node removal, we need to evaluate the second moment of the degree distribution in the new graph $E\left[\left(K^{\prime}\right)^{2}\right]$. From [11] we know that: $E\left[\left(K^{\prime}\right)^{2}\right]=E\left[K^{2}\right](1-p)^{2}+p(1-p) \bar{K}$, where $E\left[K^{2}\right]$ is the second moment of the degree distribution in the original graph. We will show the analysis referred to the quadratic cost model in Section 4.4.

\section{Network Models}

Many network topologies and the Internet, in particular, satisfy the following properties: (i) small-world property, according to which the average number of hops between each node pair is quite limited, (ii) local clustering, according to which the topology has highly connected zones, (iii) heavy-tailed distributions of the node degree, meaning that, in general, most of the nodes have few links while a few nodes have a large number of links. In the literature, several random graph models have been proposed to generate topologies matching the properties of the real ones. However, deciding which model better fits the real topology is an open problem [12]. Therefore, 
instead of focusing on a single model, we consider a few among the most popular models, showing that common properties regarding energy consumption can be inferred in all cases. In particular, we consider three well-known graph models: Erdös and Rényi, Power Law and Watts-Strogatz models.

In the Erdös and Rényi (ER) model [13] nodes are connected by links according to a given probability, and the resulting degree distribution follows a Poisson distribution. The properties of this model are well-known in the literature and have been extensively studied. In particular, the ER model exhibits the small-world property, according to which the diameter of the graph scales as $\log (N)$. However, the local clustering and heavy-tailed properties are not met.

In the Power Law (PL) model [11] the distribution $P_{K}(k)$ of the node degree $K$ follows a power-law distribution, i.e., $P_{K}(k) \sim k^{-\gamma}$. The intuition is that some nodes behave like $h u b s$, and have many more connections than others.

The Watts-Strogatz (WS) model [14] is built starting from a regular lattice in which each node is linked to a fixed number of neighbors. Then, additional edges are inserted between randomly chosen pairs of nodes ${ }^{5}$. The resulting graph is an interpolation between ordered lattices and purely random graphs. This model matches both small-world and the local clustering properties, but the degree distribution is not heavy-tailed.

In the next sections, we discuss more in detail the properties of the considered models focusing, in particular, on the first and second moments of the shortest path and the degree distributions that we need in order to compute the first and second moments of the load distribution and, hence, the energy consumption.

\subsection{The Erdös-Rényi model}

We first compute the network consumption $C$ in the case of linear cost models, i.e., the variable part of the cost model increases linearly with the load. To compute $C$ we need the expression of the average shortest path that, for an Erdös-Rényi graph, is given by [10]:

$$
\bar{d} \simeq \frac{\log (N)}{\log (\bar{K})}
$$

Using (7), (9) and (22), we can compute the all-on consumption of the network as:

$$
C=N \frac{\bar{K}}{2} F+\frac{\alpha T}{R} \frac{\log (N)}{\log (\bar{K})}+N F_{N}+2 \frac{\beta T}{R} \frac{\log (N)}{\log (\bar{K})}
$$

After randomly removing a fraction $p$ of the nodes, the average shortest path becomes: $\bar{d}^{\prime}=$ $\frac{\log (N(1-p))}{\log (K(1-p))}$ and, from (10), the network consumption becomes:

$$
\begin{aligned}
& C^{\prime}=N \frac{\bar{K}}{2}(1-p)^{2} F+\frac{\alpha T}{R} \frac{\log (N(1-p))}{\log (K(1-p))}+ \\
& N(1-p) F_{N}+2 \frac{\beta T}{R} \frac{\log (N(1-p))}{\log (K(1-p))}
\end{aligned}
$$

To compute the network consumption when the quadratic cost model is used, we need to know the second moments of both the degree and shortest path distributions, as shown in the previous section. The degree distribution in an ER graph follows a Poisson distribution, thus: $\bar{K}=\sigma_{K}^{2}$ and $E\left[K^{2}\right]=\bar{K}^{2}+\bar{K}$. For what concerns the variance of the shortest path distribution

\footnotetext{
${ }^{5}$ In the original WS model presented in [15] shortcuts are rewired from the lattice. However, the resulting graph is affected by a not negligible probability to be disconnected. Therefore, we adopt the modification of the WS model proposed by [14], in which shortcuts are additionally inserted as new links. In this way, the resulting graph is always connected.
} 
$\sigma_{d}^{2}$, it can be shown that it tends to zero when the number of nodes $N$ is high (see [16] for details). Thus, we assume that $\sigma_{d}^{2}=0$ and we obtain $E\left[d^{2}\right]=\bar{d}^{2}+\sigma_{d}^{2}=\bar{d}^{2} \approx\left(\frac{\log (N)}{\log (\bar{K})}\right)^{2}$. Finally, we can compute the network consumption using (19), (20) and (21).

\subsection{Power-law model}

We consider a graph in which the distribution of the degree $K$ follows a power law, i.e., $P_{K}(k) \sim$ $k^{-\gamma}$. In this case, the average shortest path can be computed as in [10]:

$$
\bar{d} \approx 1+\frac{\log (N / \bar{K})}{\log \left[\left(E\left[K^{2}\right]-\bar{K}\right) / \bar{K}\right]}
$$

In particular, we consider a Pareto distribution for the degree since it is one of the most widely used and studied power laws in the literature. The Pareto distribution is described by the parameters $\left(a, k_{m}\right)$, where $k_{m}$ is the minimum possible value of $K$, and $a$ is a positive parameter. For this distribution we have: $\bar{K}=a k_{m} /(a-1)$ and $E\left[K^{2}\right]=a k_{m}^{2} /(a-2)$.

Using the linear cost model, the all on network consumption $C$ can be computed similarly to what done for the ER model using (7), (9) and (25). As reported in [11], a power law graph remains power law even after a random removal of nodes. Thus, the average shortest path of the network with sleep modes is:

$$
\bar{d}^{\prime} \approx 1+\frac{\log (N / \bar{K})}{\log \left[\left(\frac{E\left[K^{2}\right]-\bar{K}}{\bar{K}}\right)(1-p)\right]}
$$

from which the network consumption with sleep mode $C^{\prime}$ can be computed.

To compute $C$ in the case of quadratic cost model, we need also to estimate the second moment of the shortest path distribution. For the PL graph we have: $\sigma_{d}^{2} \simeq \frac{2}{9} \frac{\log (N)}{\log (3)}($ see $[18])$. Thus, the second moment of the shortest path distribution is:

$$
E\left[d^{2}\right] \approx\left(1+\frac{\log (N / \bar{K})}{\log \left[\left(\frac{E\left[K^{2}\right]-\bar{K}}{\bar{K}}\right)(1-p)\right]}\right)^{2}+\frac{2}{9} \frac{\log (N)}{\log (3)}
$$

\subsection{The Watts-Strogatz model}

The Watts-Strogatz (WS) model interpolates between ordered lattices and purely random graphs [17]. Starting from a lattice of $N$ vertices in which each node is symmetrically connected to its $K_{L}$ nearest neighbors, randomness is introduced by independently adding $x$ shortcuts between randomly chosen pairs of nodes [14]. The mean node degree, $\bar{K}$, is given by $\bar{K}=K_{L}+2 x / N$.

We first focus on the computation of the network consumption when a linear cost model is used. In the literature, there are some models for the estimation of the average shortest path length when a WS model with rewiring is considered. For example, for small values of $x$, i.e., $x<<N / K_{L}$, approximation of [17] holds,

$$
\bar{d} \simeq \frac{N}{K_{L} / 2} \frac{\log (2 x)}{4 x}
$$

In this case, $\bar{d}$ is similar to the shortest path of a lattice, scaled by a factor that takes into account the number of random links. For large values of $x$, i.e., $x>>N / K_{L}$, the WS graph with rewiring 
is similar to a purely random graph [14] and the average shortest path can be approximated as,

$$
\bar{d} \simeq \frac{\log (N)}{\log (\bar{K})}
$$

However, both approximations do not hold in our case, since we are using the WS model without rewiring, i.e. we add the random links to the graph, and the number of random links is quite large. We therefore propose a model that fits the scenarios we are interested in. By considering a large number of simulation results for cases of interest to this study, we have derived the following expression to compute $\bar{d}$ :

$$
\bar{d} \approx \frac{N}{K_{L} / 2} \frac{\log (2 x)}{4 x}+\frac{\log (N)}{\log (\bar{K})}
$$

Intuitively, we have found that in our scenarios $\bar{d}$ scales like a random graph plus the corrective term of $(28)$.

During the sleep mode regime, the average shortest path becomes:

$$
\bar{d}^{\prime} \approx \frac{N}{K_{L} / 2} \frac{\log \left(2 x(1-p)^{2}\right)}{4 x(1-p)^{2}}+\frac{\log (N(1-p))}{\log (\bar{K}(1-p))}
$$

Similarly to the previous graph models, using (12), (30) and (31), we derive the cost $C$ and $C^{\prime}$ for the WS graph in this regime.

To evaluate the total cost with a quadratic cost model we also need the second moment of the shortest path and the degree distributions of a WS graph. We propose the following new model for $E\left[d^{2}\right]$ that is derived by interpolation from simulation results:

$$
E\left[d^{2}\right] \approx\left(\frac{\log (N)}{\phi^{\tau} \log \left(K_{L}\right)+\log \left(\frac{2 x}{N}\right)}\right)^{2}+\chi
$$

with $\phi=\frac{2 x / N}{K_{L}}, \tau=0.08$ and $\chi=15$.

For what concerns the degree distribution, we use $E\left[K^{2}\right] \approx \bar{K}^{2}+K_{L}$, assuming a behavior similar to a ER graph.

\subsubsection{Model Validation}

We validated the proposed WS model by comparison against simulation results in a large number of scenarios. We consider networks with a given value of $N$ and of the constant part of the neighbor degree, $K_{L}$, but different values of $x$. By varying $x$ we make the random component of the degree vary.

Top plot of Fig. 2 reports the average shortest path $\bar{d}$ for the case $N=10,000, K_{L}=4$, and $x \in[5,000-20,000]$. For each value of $x$, the results are averaged over 20 independent runs in which different random seeds are used for adding the shortcuts. The figure reports $\bar{d}$ computed from: (28) that corresponds to the model of a scaled lattice (SL label in the figure), (29) that is the ER model, our proposed model (30), and simulation results. Clearly, the SL model does not match the measured $\bar{d}$ for the considered scenarios; the ER model underestimates $\bar{d}$, since it does not consider the presence of many links to neighbors. Our model presents a good matching, since it is fitted for these scenarios. We have also validated the model in other scenarios, including scenarios with sleep modes, obtaining similar results.

Bottom plot of Fig. 2 shows the energy reduction ratio, $E$, computed by simulation and with the proposed model, for the case of a network with sleep modes, $N=10,000$ nodes, $K_{L}=4$, 

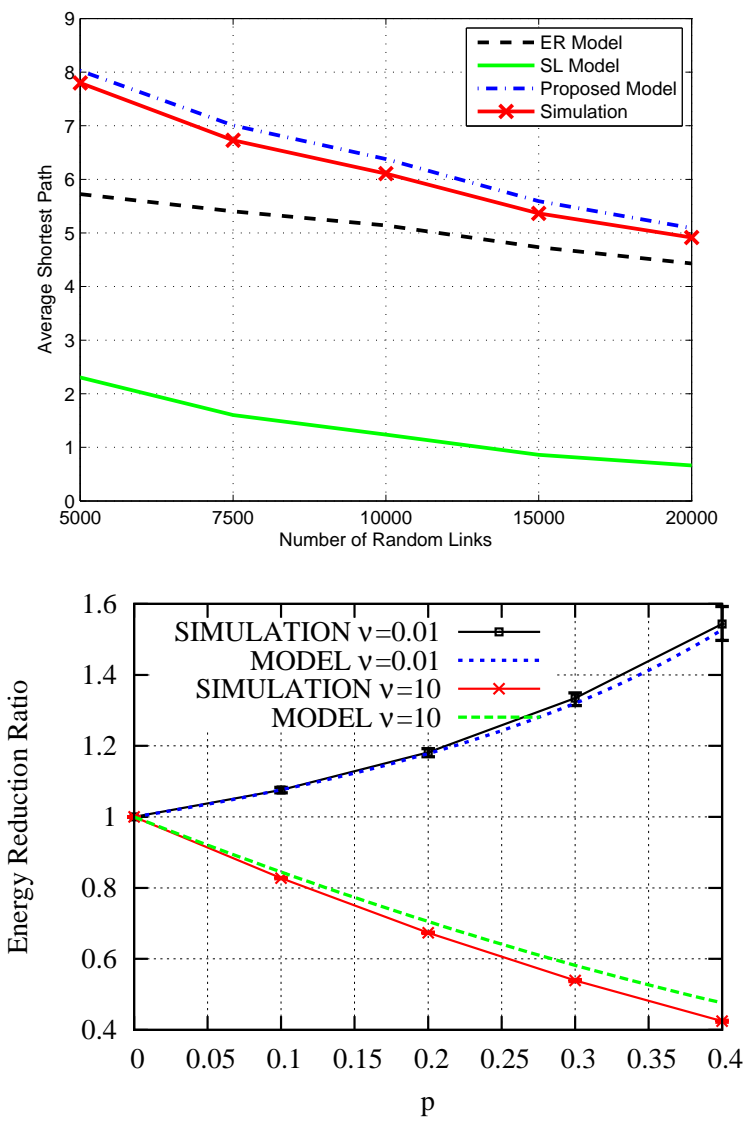

Figure 2: Validation of the proposed model to compute $\bar{d}$ for WS graphs: (top) average shortest path for different models and by simulation, considering $N=10,000, K_{L}=4, x \in[5,000-$ 20,000], (bottom) energy reduction ratio versus $p$ for the proposed model and the simulation, considering $N=10,000, K_{L}=4, x=10,000$.

$x=10,000$ and $\nu=\nu_{N}$; the fraction of nodes that are switched off varies between 0 and 0.4. Bars indicate minimum and maximum values of the energy reduction ratio. Moreover, we have also computed the confidence intervals of $E$, finding that they never exceed $1 \%$ of the estimated value at $95 \%$ confidence level. Again, observe how accurate the proposed model is.

We then validate the WS model also for the case of quadratic cost function. Top plot of Fig. 3 reports $E\left[d^{2}\right]$ for $N=10,000, K_{L}=4$, and $x \in[5,000-20,000]$, considering the ER model, the simulations, and the proposed model of (32). The lattice model (not reported in the figure) highly overestimates $E\left[d^{2}\right]$, with values in the order of $10^{6}$. Our model presents a very good matching with the simulation results.

Bottom plot of Fig. 3 reports the energy reduction ratio $E$ for $N=10,000$ nodes, $\bar{K}=6$, $x=10,000$ and $p \in[0,0.4]$. In this case the confidence intervals of $E$ never exceed $5 \%$ of the estimated value at $95 \%$ confidence level. Our proposed model matches the simulated $E$ considering different values of $\nu$. In the following we therefore adopt our models for computing $C$ and $C^{\prime}$. 

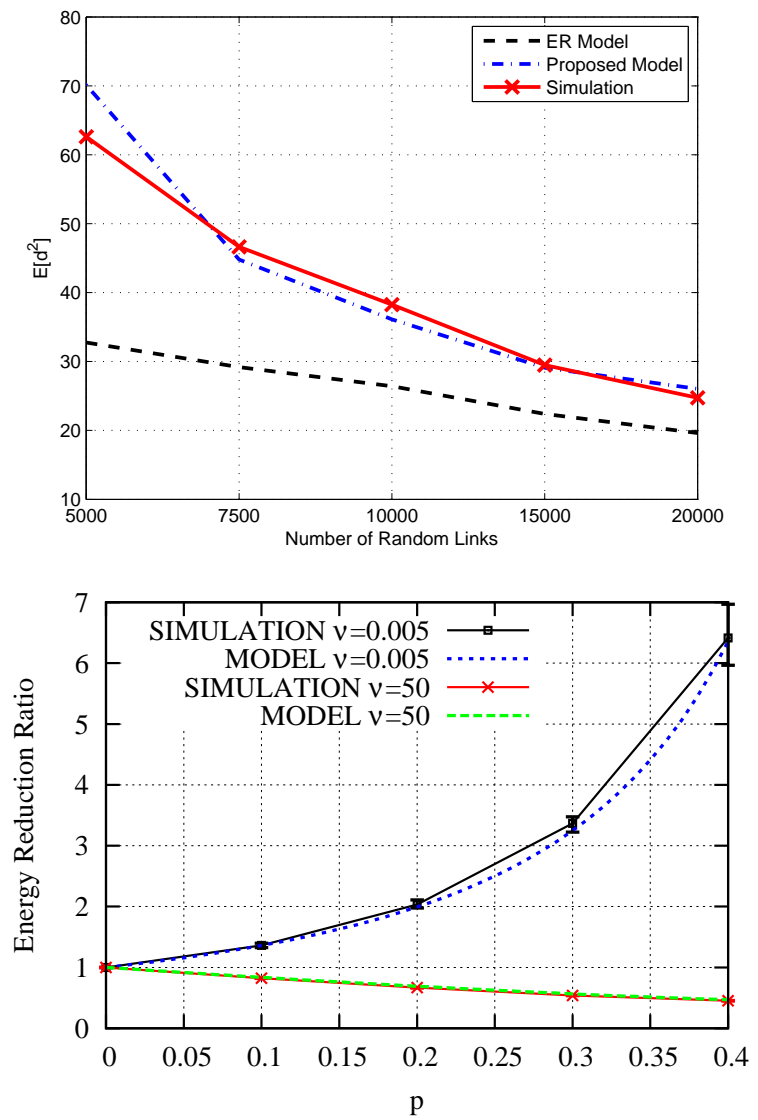

Figure 3: Validation of the proposed model for the quadratic load function for WS graphs: (top) $E\left[d^{2}\right]$ for different models and by simulation, considering $N=10,000, K_{L}=4, x \in[5,000-$ 20,000], (bottom) energy reduction ratio versus $p$ of different models considering $N=10,000$, $K_{L}=4, x=10,000$.

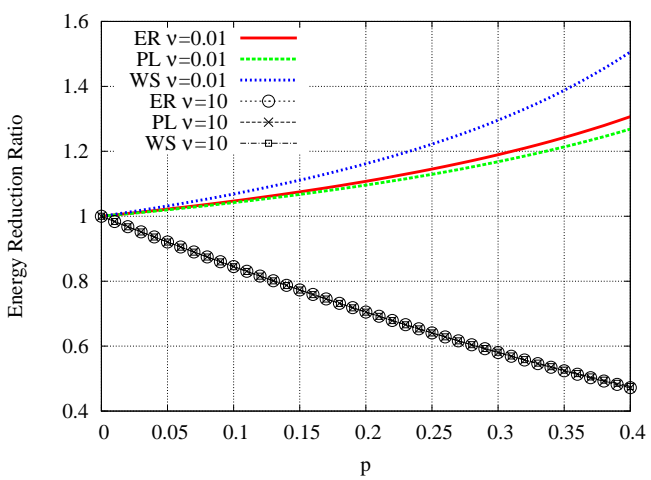

Figure 4: $E$ versus $p$ for ER, PL and WS graph models. 

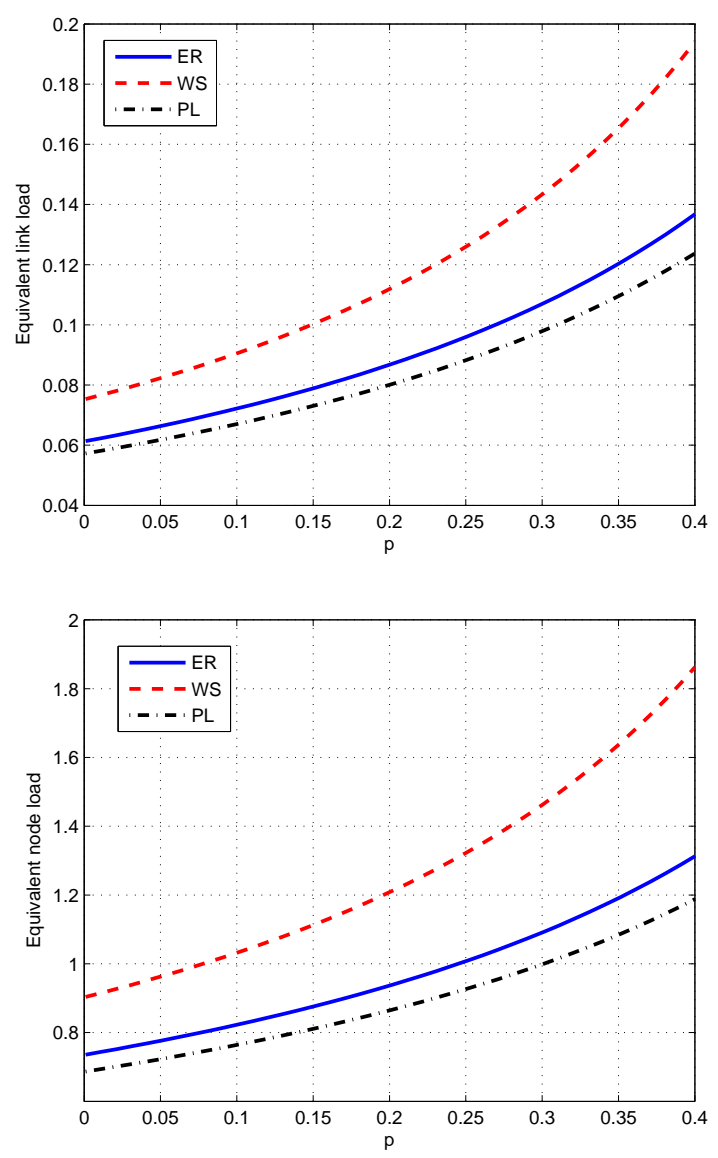

Figure 5: Equivalent link load $\nu$ (top) and equivalent node load $\nu_{N}$ (bottom) versus the switch-off probability $p$ for the ER, PL and WS model. 

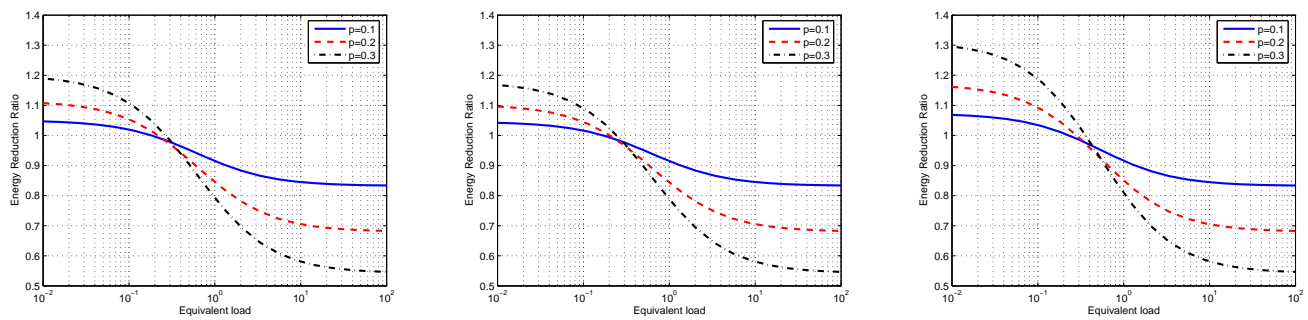

Figure 6: Energy reduction ratio $E$ versus equivalent load $\nu$ for different values of $p$, from left to right: ER, PL, WS model.

\section{Performance Evaluation}

In this section, we compare the effectiveness of the approaches based on sleep modes under the different network models proposed in the previous section.

For the numerical results, unless otherwise specified, we set $N=10,000$ and the link rate $R=100 \mathrm{Gbps}$. The link and node linear power model of (4) and (6) is used. The average degree $\bar{K}$ is set to 6 . This reflects the results of recent measurement studies about actual network topologies like the Internet (see [19] for an overview), according to which $\bar{K}$ ranges between 4 and 8. We assume that the total traffic scales with the number of nodes $N$ and the degree $\bar{K}$. Notice also that we impose an amount of traffic that does not overload the network when the maximum amount of devices is switched off, i.e., $\bar{\rho}^{\prime}<1$ for $p=0.4$.

For the PL model, we choose a Pareto distribution of the node degree, setting $a=3$ and $k_{m}=4$, so that $\bar{K}=6$; for the WS model, we set $K_{L}=4$ and $x=10,000$ and the average node degree is $\bar{K}=K_{L}+2 x / N=6$.

\subsection{Impact of Sleep Modes}

We first evaluate the impact of sleep modes on energy consumption for different graph models. Fig. 4 reports the energy reduction ratio versus $p$ for two distinct cases: high equivalent load $\left(\nu=\nu_{N}=10\right)$ and low equivalent load $\left(\nu=\nu_{N}=0.01\right)$. When the constant part of power is predominant $(\nu=10)$ the introduction of sleep modes saves energy in the network, i.e., $E<1$, for all the graph models. Moreover, the energy reduction ratio decreases as $p$ increases, suggesting that the saving increases with the number of devices that are switched off. Notice also that the curves of WS, PL and ER are overlapping since the constant part of power is the same for the three graph models, while the impact of the variable power is negligible. On the contrary, when highly energy-proportional networks are considered $(\nu=0.01)$, the network consumes a higher amount of energy when devices are switched off and $E>1$. Thus, in this scenario, sleep modes are not convenient. In particular, $E$ is higher for WS than for ER and PL models, due to the fact that, in the WS graph model, node removal has an important impact on the shortest paths.

We then analyze the impact of sleep modes by separately analyzing the impact of links and nodes power consumption. In particular, from (14) and (15) we compute the minimum values of $\nu$ and $\nu_{N}$ for which $E$ becomes smaller than 1; these values represent breakeven points for which sleep mode saves energy. The top (bottom) plot of Fig. 5 reports the breakeven curve for links (nodes). If the value of $\nu$ falls above the breakeven curve, sleep mode is convenient; otherwise, the network consumes a higher amount of energy when devices are switched off. In all cases, as $p$ increases the minimum value of $\nu$ increases too, meaning that for large values of $p$ 

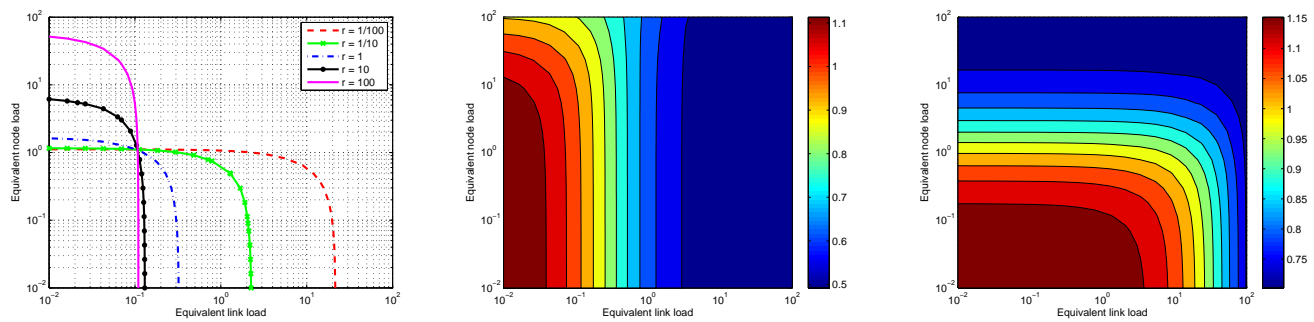

Figure 7: ER model: (left) breakeven curves corresponding to $E=1$ versus $\nu$ and $\nu_{N}$, (central) energy reduction ratio for $r=10^{2}$, (right) energy reduction ratio for $r=10^{-2}$.

a sleep mode approach is convenient only when the constant part of the energy cost is high with respect to the variable part. In particular, from the top plot of Fig. 5, it can be observed that the breakeven curve of the ER model ranges between 0.06 and 0.14 . The WS breakeven curve is above the other two curves, due to the fact that the average shortest path length increases faster for WS (and load increase is thus more significant) than in the other models; or, in other terms, even with devices that present quite significant load proportionality, sleep modes are convenient. From bottom plot of Fig. 5, it can be observed that the breakeven curves of nodes are one order of magnitude larger than the corresponding ones of links. This is due to the fact that the variable term of nodes power grows much faster than the links one, thus higher fixed costs are required to balance the increase in the node load.

\subsection{Impact of Technology Constraints}

We now evaluate the impact of the equivalent load. Fig. 6 reports the values of $E$ versus $\nu$ for the three proposed models; the different curves correspond to different values of $p$ where for simplicity we set $\nu=\nu_{N}$. In all cases, the breakeven point for which $E=1$ occurs when the fixed cost $F\left(F_{N}\right)$ is one order of magnitude lower than $\alpha(\beta)(\nu \approx 0.3)$. Two different regimes are possible: i) sleep mode is not convenient $(E>1)$, and ii) sleep mode is convenient $(E<1)$. In the first regime, the higher the probability to switch off devices is, the higher the additional energy is, being WS the worst case. In the second regime, instead, sleep mode leads to high energy saving for all models, and the saving strongly increases with $p$. Notice that with today technology, we are in the right part of the figures (sleep mode is always convenient, with saving up to $30-40 \%$ ), while in the future, the values of $\nu$ will probably decrease, meaning that sleep mode will become less convenient.

In the following, we consider the impact of varying the relative cost of nodes and links. In particular, we introduce a scaling factor $r$ for the cost of nodes, so that the total network $\operatorname{cost} C$ becomes:

$$
C=\sum_{j \in \mathcal{L}} A(j)+\frac{1}{r} \sum_{i \in \mathcal{N}} A_{N}(i)
$$

In this way, we find the tradeoffs that emerge as the costs of nodes and links are weighted differently. For example, the cost of links is normally marginal for wireless technologies: the largest amount of power is needed to keep powered on Base Stations in UMTS or Access Points in WiFi networks [26]. On the contrary, for backbone networks the cost of links is not negligible, due to the presence of multiple amplifiers for optical networks or DSL lines for home access networks [27]. 


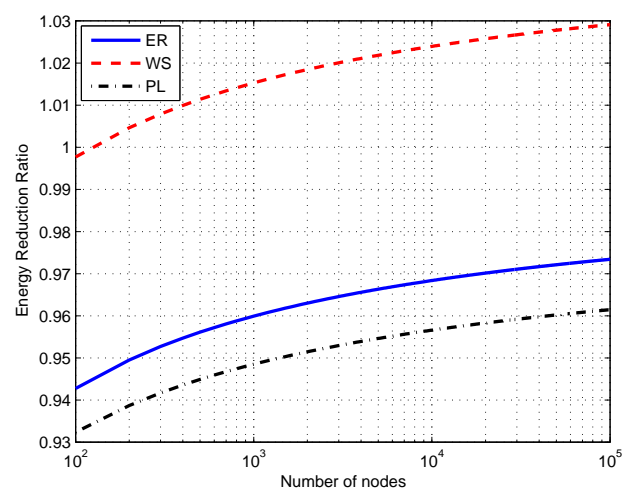

Figure 8: Energy reduction ratio $E$ versus the number of nodes $N$ for $\nu=0.5$ and $p=0.3$.

Left plot of Fig. 7 reports the breakeven curves for which $E=1$ varying both $\nu$ and $\nu_{N}$, considering different values of the parameter $r$. In this case, we are considering an ER graph model and $p=0.3$. Points below a breakeven curve correspond to conditions in which sleep modes are not convenient. For low values of $\nu$ and $\nu_{N}$ sleep modes are not convenient, i.e., $E>1$ (bottom left corner of the plot). Conversely, for high fixed costs (top right corner of the plot) sleep modes are convenient, $E<1$. As $r$ increases, the network becomes almost insensitive to nodes equivalent load. For example, for $r=100$, sleep modes are convenient if $\nu>0.12$ or $\nu_{N}>40$. On the contrary, for $r=10^{-2}$ sleep modes are convenient if $\nu>20$ or $\nu_{N}>1$.

To give more insights, central and right plots of Fig. 7 detail the energy reduction ratio for $r=10^{2}$ and $r=10^{-2}$, respectively. The vertical colorbars report the values of the energy reduction ratio. As expected, for $r=10^{2}$ the energy reduction ratio mainly depends on the value of $\nu$. Notice that for $\nu>>1$ (i.e., $F>>\alpha$ ), the energy reduction ratio of the network approaches the limit $(1-p)^{2}=0.49$ obtained when $\alpha=0$. Interestingly, for $r=10^{-2}$ (right plot) the energy reduction ratio still depends on the cost of the links, so that the network saves up to $25 \%$ of energy when $\nu=100$, independently of $\nu_{N}$. This is due to the fact that, for a given $p$, the fraction of links powered off is always larger than the fraction of nodes.

\subsection{Impact of Network Properties}

We now consider the impact of the network properties on the possible energy saving. In particular, we start by setting $\bar{K}=6$, while we vary $N$ in $\left[10^{2}, 10^{5}\right]$. For the WS model we set $x=N$. Fig. 8 reports the energy reduction ratio $E$ for the considered models, for $\nu=\nu_{N}=0.5$ and $p=0.3$. The highest saving is obtained by the PL model. In all the cases, $E$ increases (sleep mode effectiveness reduces) with the number of nodes; this is due to the fact that we increase $N$ while letting the node degree unchanged, so that the set of alternative paths does not scale with $N$ and the impact of switching off some devices on the path length increases. This is particularly visible under the WS model.

Now we consider the impact of the average node degree $\bar{K}$ and select values of $\bar{K}$ that mimic those used in [19] to represent the average node degree of large topologies. We set $\nu=\nu_{N}=0.5$ and $p=0.3$. Moreover, for the WS model we fix $x=10,000$ and we vary $K_{L} \in[2,8]$. Fig. 9 shows $E$ versus $\bar{K}$. For all the models, the energy reduction ratio is decreasing as $\bar{K}$ is increasing. Indeed, for large values of $\bar{K}$, the number of possible paths between any two nodes is large and, once some nodes are powered off, it is easy for the network to find alternative paths; or, in other 


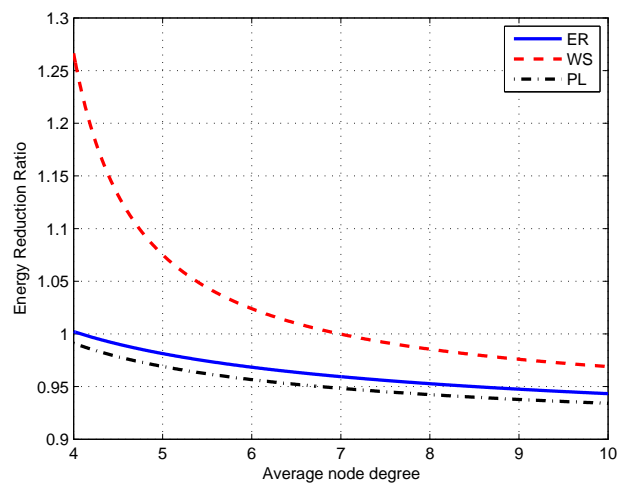

Figure 9: Energy reduction ratio $E$ versus average node degree $\bar{K}$ for $\nu=0.5$ and $p=0.3$.

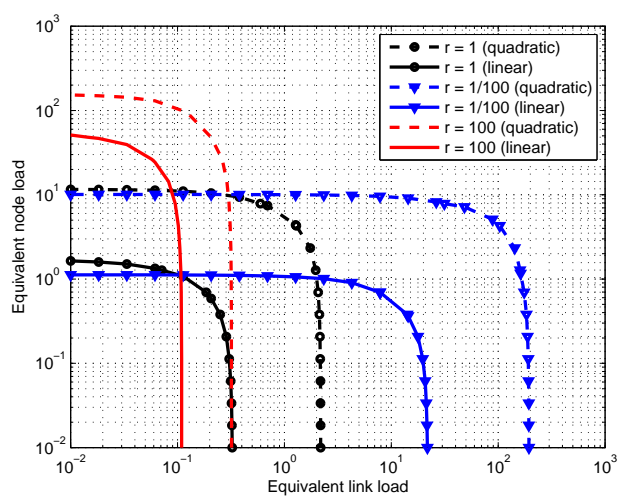

Figure 10: ER quadratic-linear comparison for $p=0.3$.

terms, the increase of the average shortest path due to sleep modes is limited if $\bar{K}$ is large. For low values of $\bar{K}$, sleep modes are not convenient. For example, for the WS model $E$ is around 1.27 when $\bar{K}=4$, meaning that the sleep mode wastes an additional $27 \%$ of power with respect to an always on solution. Conversely, when $\bar{K}$ increases, the additional cost due to the increase of the path length is smaller, so that sleep modes are more efficient. Finally, notice that the energy reduction ratio of the ER and PL models is consistently lower than the WS one. This is due to the better path length properties the former two graphs have.

\subsection{Impact of Power Consumption Model}

We consider here the impact of the power consumption model. In particular, we compare the linear power consumption model of (7) and the quadratic model of (19). Fig. 10 details the breakeven curves for which $E=1$, comparing the two models for different values of $r$ under the ER network model. Interestingly, the area for which $E>1$ steadily increases with the quadratic model, since the weight of the variable term is higher than the linear case.

We then consider the impact of the quadratic cost function when the fraction of switched off nodes varies. Fig. 11 shows the energy reduction ratio versus the probability to remove nodes $p$, 

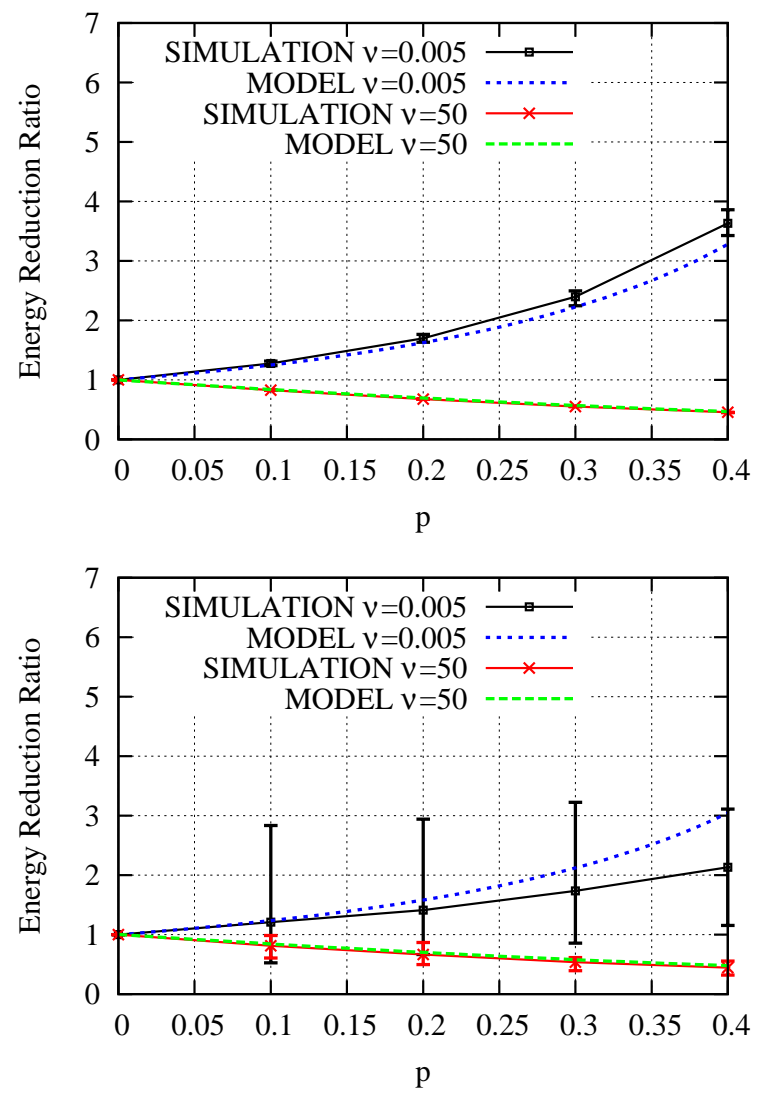

Figure 11: Energy reduction ratio versus $p$ with the quadratic cost model: ER model (top) and PL model (bottom).

for the ER and PL graph models (refer to Fig. 3 for the WS model). In this case, we consider $\nu=\nu_{N}=0.005$ and $\nu=\nu_{N}=50$. The figures report also the average results obtained by simulation. Bars indicate minimum and maximum values. Interestingly, when $\nu=0.005$ the variable term of power is predominant and $E>3$ for $p=0.4$ considering the ER model. This means that a strongly energy proportional network with sleep modes can consume up to three times an all-on network. Notice also that, with the same parameters, the WS model performs consistently worse, while the PL performs quite better. On the contrary, as high fixed costs are considered, all the models save energy, being $E<1$ for all values of $p$. Finally, all the models present a good matching with the simulations, indicating that the introduced approximations do not impact on the variation of $E$.

\section{Comparison of Switching Policies}

Finally, we investigate the case in which the nodes to be switched off are chosen according to another strategy than a simple random removal policy. Note that, the choice of the node removal strategy is out of the main scope of this paper. Nevertheless, in the following we compare the simple random removal strategy with the least-flow policy of [4] to assess the effectiveness of sleep 

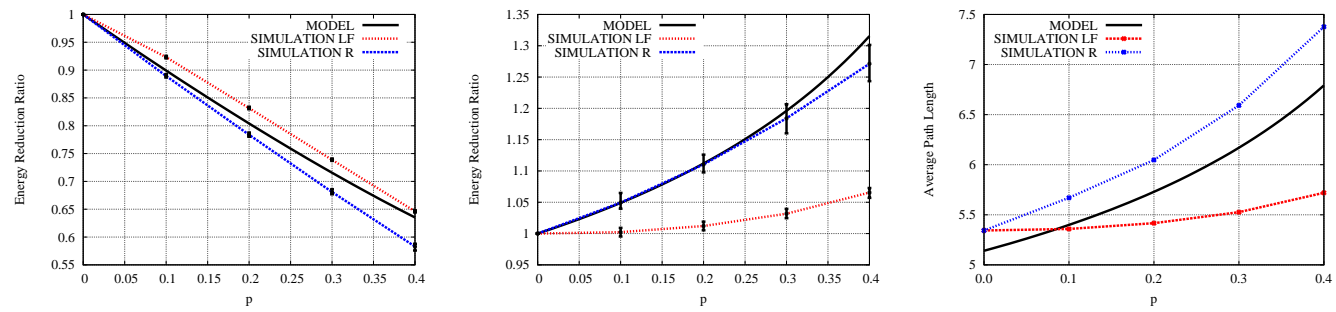

Figure 12: Least-Flow (LF) versus Random (R) policy comparison for the ER graph model: energy reduction ratio versus $\mathrm{p}$ for $\nu=10$ (left) and $\nu=0.01$ (center); average path distance (right).

modes under a smart switch-off policy. Specifically, the least-flow strategy consists in switching off the devices with the least amount of traffic flowing through them, where the total amount of flow crossing a node is computed as the sum of the traffic flowing through its incident links. This strategy could be particularly effective in reducing the rerouted traffic in the network when a node is powered off, in order to maintain an adequate QoS for users. Note that our models apply only to random strategy, thus, the results of the least-flow policy are derived by simulation.

Fig. 12 shows the energy reduction ratio versus $p$ for the random $(\mathrm{R})$ and least-flow (LF) policies considering the ER graph model and $r=1 / 10$. Several considerations hold in this case. First, with little proportional networks (left plot) the energy reduction ratio of the least-flow policy is higher than a pure random policy. This is due to the fact that most of least loaded nodes have low degree, thus the number of actually switched off links is lower than in the random case, in which a fraction $1-(1-p)^{2}$ of links is switched off. Second, as predominant variable terms of power are considered (central plot), the energy reduction ratio of the least-flow policy is consistently lower than the random policy. This is due to the fact that the average path length after the nodes removal grows slower than in the random policy, since the least loaded devices carry less traffic. This is confirmed by the right plot of the same figure that reports the average path length in the considered cases.

\section{Discussion}

In this section we briefly discuss the main assumptions of our model and how much the results are impacted by these assumptions.

Network Topology. In the literature, the effectiveness of sleep modes has been evaluated on benchmarking topologies (see for example [4] and [22]). However, the obtained results are tailored to specific reference topologies, and are difficult to be generalized. In this work, we follow a different approach: we exploit graph theory to model network topologies, and we derive general insights, showing what happens when network parameters, such as the number of nodes and the average node degree, are varied. In this way, we are able to overcome the limitations derived from the analysis over a specific topology. However, we recognize that actual topologies can be more complex than a single graph model. To partially cope with this issue, rather then showing results for a single model, in this work we have considered different graph models, showing that common properties can be inferred in all cases.

Set of Devices in Sleep Mode Another crucial aspect is how to choose the set of devices to be put in sleep mode. In the literature, several algorithms have been proposed to select the set of devices to be powered off (see for example [4, 22, 23, 24]). In this work, we assume that the 
set of devices in sleep mode is given and is such that the minimum required QoS is guaranteed to all the users. For analytical tractability, we adopt the conservative assumption that the devices in sleep mode are randomly chosen. We recognize that the actual savings may be even larger if smarter algorithms are considered. However the most interesting finding of the paper, i.e., the fact that sleep mode is effective even when load proportional devices are deployed, is still valid and general. To further support this insight, in this paper we compare the random policy with the least-flow policy of [4], which is more effective in choosing the set of devices in sleep mode. We show that the general message still holds even when the this advanced policy is adopted.

Traffic Variation. In a telecommunication network traffic varies over time, suggesting that sleep mode can be dynamically applied in order to follow the traffic trend [28], [29]. In this work, we consider a snapshot of the network at a given time, and evaluate the effectiveness of sleep mode for the selected snapshot. Our model gives indications for the considered scenario. Finally, note that transient behavior of devices and of the whole network has to be considered. This includes the latency introduced to recover from a sleep mode state, and how to react to sudden load fluctuations. These aspects are out of the scope of this paper.

Traffic Distribution. Our framework takes as input the total amount of traffic exchanged in the network, which we assume to be uniformly distributed among all nodes. If a real traffic matrix shows unbalanced traffic demands, e.g., a peering point collecting most of the traffic in the network [4], a uniform matrix is a conservative scenario, since many devices are required to be at full power. This intuition has been confirmed in previous work too [4].

Power and Capacity Distribution. We assume that all the devices (nodes or links) consume the same power. This assumption can be considered representative of current core networks, in which all devices have a similar capacity [4]. Extensions to classes of devices are possible, and we leave it for future work. Considering power consumption model of devices, several measurements corroborate the simple linear relationship with offered traffic. More advanced scaling mechanisms (such as DVS) are also modeled by quadratic functions. Extensions to higher degree polynomial functions are possible, but not straightforward.

\section{Related Work}

The study of power-saving network devices has been introduced in recent years, starting from the pioneering work of [30]. Rate adaptation and sleep mode techniques have recently attracted the attention of the research community (see [33, 34] for an overview). In [35] authors focus on two power management schemes for power saving, based either on sleeping or rate adaption. Differently from our work, they consider to put into standby mode network elements during idle times, i.e., among subsequent packet arrivals. In our work, instead, we assume that devices are put in sleep mode over coarser time scales, i.e., of the order of minutes and hours. Moreover, they consider the impact of the two techniques in isolation, while in our work we assess the impact of sleep mode over a variety of scenarios, including energy-proportional networks.

Moreover, in the last years, Dynamic Voltage Scaling techniques that dynamically adjust the frequency and voltage of links have been shown to reduce power consumption [31]. However, these techniques require sophisticated hardware mechanisms to ensure correct link operation during scaling, and the potential power savings can be smaller than the ones achievable with sleep modes since links are still powered on even when they are in an idle state. Furthermore, the authors of [32] investigated the savings achievable dynamically turning links on/off in response to traffic variation. However, their work is targeted to a rather different field, i.e., the interconnection network fabrics. In particular, the topology of an interconnection network is represented by a grid, while an ISP network has a less regular structure. Moreover, the authors of [32] consider 
only the case in which links only are put in sleep mode and their analysis is limited to a simple power model on/off, whithout investigating the case in which energy-proportional devices are used.

In [36] authors estimate the total power consumption of a telecommunication network, given the power requirements of network devices and the traffic from users. In particular, they consider all the network portions of a telecom operator, foreseeing that the highest energy consumption growth rates will be in the data centers and IP backbone networks. Basing their assumptions on technology forecasts, they claim that future networks with enabled sleep modes and load adaptation will save a consistent amount of energy, i.e., typically larger than $56 \%$. While this intuition is inline with our results, our model is able to derive more general insights, being able to highlight the breakeven points that emerge adopting different power models and technology assumptions.

Power consumption of current network devices is mostly independent of the current load, considering backbone [1,5] and access networks [26]. Moreover, the power consumption of links is not negligible, and can be of the same order of magnitude of nodes [27]: this is especially true for long-haul optical links, in which several amplifiers are needed to regenerate the signal. Step functions are adopted to model power consumption of such devices. Several works have therefore targeted the minimization of network devices powered on while satisfying QoS [25, 33, 34]. Our work confirms that, in this scenario, networks with sleep nodes are the best solution in terms of power saving.

Researchers from universities and industries are now studying solutions to adapt power with current load. In particular, in [7] the authors exploit the idea of exchanging energy profiles among devices to reduce the overall power consumption during routing and traffic-engineering operations. They consider different models to represent device power consumption, including linear and super-linear models. In [8] authors evaluate the impact of sleep modes over different network topologies. They consider different power models, evaluating the total power consumption when some elements are put into sleep modes. All these works are mainly focussed on specific case-studies, while our framework is able to produce more general insights over a variety of network graphs, showing that the gain adopting sleep modes can be consistent even when energy-proportional devices are deployed.

Overall, the consciousness of power-aware telecommunication networks is growing. Several projects are investigating the impact of power adaptive technologies for network devices [37, $38,39,40]$. Indeed, this work is undertaken under the project TREND (Towards Real Energyefficient Network Design) [41], a Network of Excellence funded by the European Commission through the FP7 Program.

\section{Conclusions}

In this paper, we have proposed an analytical framework for the evaluation of the potential energy saving that can be achieved by applying sleep modes to the devices of a complex network, like the Internet. We have modeled the network device (nodes and links) power consumption by means of a simple function composed of a constant cost and a variable cost proportional to the device load. Leveraging on random graph theory, we have then computed the overall power consumption of networks with different topological properties. Random graph theory results are used to evaluate the total power consumption of a network with all the devices powered on, or a fraction of devices only. The comparison of these figures has enlightened when the sleep mode adoption is convenient.

Our results suggest that with today technology, with device consumption that varies very 
little with the load, the use of sleep modes is very effective in reducing the network energy consumption. In the future, with devices whose consumption will likely be more load proportional, the effectiveness of sleep mode approaches will reduce. However, we have found that sleep modes reduce power consumption even when device power scales quadratically with load. Our results indicate also that highly connected networks, with large node degree and high randomness, tend to make the use of sleep modes more convenient.

\section{Acknowledgment}

This work received funding from the European Commission Seventh Framework Programme under grant agreement 257740 (Network of Excellence TREND).

\section{References}

[1] A. Adelin, P. Owezarski, and T. Gayraud, "On the Impact of Monitoring Router Energy Consumption for Greening the Internet," IEEE/ACM International Conference on Grid Computing (Grid 2010), Bruxelles, Belgique, October 2010.

[2] R. Bolla, R. Bruschi, K. Christensen, F. Cucchietti, F. Davoli; S. Singh, "The Potential Impact of Green Technologies in Next-Generation Wireline Networks - Is There Room for Energy Saving Optimization?," IEEE Communication Magazine (COMMAG), 2011.

[3] L. A. Barroso, U. Holzle, "The Case for Energy-Proportional Computing," IEEE Computer, V. 40, N. 12, pages 33-37, December 2007.

[4] L. Chiaraviglio, M. Mellia, F. Neri, "Minimizing ISP Network Energy Cost: Formulation and Solutions," IEEE/ACM Transactions on Networking, July 2011.

[5] P. Mahadevan, P. Sharma, S. Banerjee, and P. Ranganathan, "A power benchmarking framework for network devices," NETWORKING, 2009.

[6] A. Francini, D. Stiliadis, "Rate adaptation for energy efficiency in packet networks," Bell Labs Technical Journal, Special Issue: Green Information and Communications Technology (ICT) for Eco-Sustainability, V. 15, N. 2, pages 131-146, September 2010.

[7] J. Restrepo, C. Gruber, C. Machoca, "Energy Profile Aware Routing," IEEE GreenComm 09, Dresden, Germany, June 2009.

[8] S. Antonakopoulos, S. Fortune, L. Zhang, "Power-aware Routing with Rate-adaptive Network Elements", IEEE Globecom 2010 Workshop on Green Communications, Miami, USA, December 2010.

[9] L. Chiaraviglio, D. Ciullo, M. Mellia, M. Meo, "Modeling Sleep Modes Gains with Random Graphs", IEEE INFOCOM 2011 Workshop on Green Communications and Networking, Shanghai, China, April 2011.

[10] R. Pastor-Satorras, A. Vespignani, Evolution and structure of the internet: A statistical physics approach, Cambridge University Press, 2004.

[11] R. Albert, and A.-L. Barabási, "Statistical mechanics of complex networks", Rev. Mod. Phys. 74, pp. 47-97, 2002. 
[12] H. Haddadi, G. Iannaccone, A. Moore, R. Mortier, and M. Rio, "Network topologies: Inference, modelling and generation", IEEE Communications Surveys and Tutorials, V. 10, N. 2,2008 .

[13] P. Erdös and A. Rényi, "On random graphs", Publicationes Mathematicae, V.6, pp. 290-297, 1959.

[14] M. E. J. Newman and D. J. Watts, "Renormalization group analysis of the small-world network model", Phys. Lett. A 263, pp. 341-346, 1999.

[15] D.J. Watts and S.H. Strogatz "Collective dynamics of small-world networks", Nature, V. 393, N. 6684, pp. 440-442, 1998.

[16] V. Blondel, J.L. Guillaume, J. Hendrickx, R. Jungers, "Distance distribution in random graphs and application to network exploration", Phys. Rev. E 76, 066101, 2007.

[17] M. E. J. Newman, C. Moore, and D. J. Watts, "Mean-field solution of the small-world network model", Phys. Rev. Lett. 84, pp. 3201-3204, 2000.

[18] S.N. Dorogovtsev, A.V. Goltsev, J.F.F. Mendes, "Pseudofractal Scale-free Web", Phys. Rev. E 65, 066122, 2002.

[19] H. Haddadi, D. Fay, A. Jamakovic, O. Maennel, A. W. Moore, R. Mortier, and S. Uhlig, "On the importance of local connectivity for Internet topology models", 21st International Teletraffic Congress, Paris, France, September 2009.

[20] Orbis topology, "http://sysnet.sysnet.ucsd.edu/sysnet/."

[21] Geant topology, "http://www.geant.net/pages/home.aspx."

[22] F. Idzikowski, S. Orlowski, C. Raack, H. Woesner, A. Wolisz, "Saving energy in IP-overWDM networks by switching off line cards in low-demand scenarios," Conference on Optical Network Design and Modeling (ONDM10), Kyoto, Japan, February 2010.

[23] A. Coiro, M. Listanti, A. Valenti, F. Matera, "Reducing power consumption in wavelength routed networks by selective switch off of optical links," IEEE Journal of Selected Topics in Quantum Electronics, vol. 17, no. 2, pp 428-436, 2011.

[24] W. Fisher, M. Suchara, J. Rexford, "Greening Backbone Networks: Reducing Energy Consumption by Shutting Off Cables in Bundled Links," in Proceedings of 1st ACM SIGCOMM workshop on green networking, New Delhi, India, August 2010.

[25] R. Bolla, R. Bruschi, A. Cianfrani, M. Listanti, "Enabling Backbone Networks to Sleep," IEEE Network, V.25, N.2, pp. 26-31, 2011.

[26] S. Chiaravalloti, F. Idzikowski, and L. Budzisz, "Power consumption of WLAN network elements," TKN Technical Report Series TKN-11-002, Telecommunication Networks Group, Technical University Berlin, August 2011. http://www.tkn.tuberlin.de/publications/papers/TR-WLAN-Power.pdf

[27] F. Idzikowski, "Power consumption of network elements in IP over WDM networks," TKN Technical Report Series TKN-09-006, Telecommunication Networks Group, Technical University Berlin, July 2009. http://www.tkn.tuberlin.de/publications/papers/powerNumbers_final.pdf 
[28] M. Gupta, S. Singh, "Using Low-Power Modes for Energy Conservation in Ethernet LANs," Proc. 26th IEEE Conf. on Computer Communications (INFOCOM 2007), Anchorage, Alaska, May 2007.

[29] S. Herrería Alonso, M. Rodríguez Pérez, M. Fernández Veiga, and C. López García, "Opportunistic power saving algorithms for Ethernet devices," Computer Networks, vol. 55, no. 9, pp. 2051-2064, Jun. 2011.

[30] M. Gupta, S. Singh, "Greening of the Internet," Proceedings of ACM SIGCOMM, Karlsruhe, Germany, August 2003.

[31] L. Shang, L.S. Peh, and N.K. Jha, "Power-efficient interconnection networks: Dynamic Voltage Scaling with links," Computer Architecture Letters, Vol. 1, No.2, May 2002.

[32] V. Soteriou and L.S. Peh, "Dynamic power management for power optimization of interconnection networks using on/off links," in Proceedings of the High Performance Interconnects, pp. 15-20, 2003.

[33] R. Bolla, R. Bruschi, F. Davoli, and F. Cucchietti, "Energy Efficiency in the Future Internet: A Survey of Existing Approaches and Trends in Energy-Aware Fixed Network Infrastructures", IEEE Communication Surveys and Tutorials, 2011.

[34] A. Bianzino, C. Chaudet, D. Rossi, and J. Rougier, "A Survey of Green Networking Research", IEEE Communication Surveys and Tutorials, 2012.

[35] S. Nedevschi, L. Popa, G. Iannaccone, S. Ratnasamy, D. Wetherall, "Reducing Network Energy Consumption via Sleeping and Rate-Adaptation", USENIX/ACM NSDI 08, San Francisco, USA, April 2008.

[36] C. Lange, D. Kosiankowski, R. Weidmann, and A. Gladisch, "Energy Consumption of Telecommunication Networks and Related Improvement Options", IEEE Journal of Selected Topics in Quantum Electronics, V. 17, N. 2, pp.285-295, April 2011.

[37] ECONET Project, "http://www.econet-project.eu."

[38] GreenTouch Consortium, "http://www.greentouch.org/."

[39] D-Link Green Products, "http://www.dlinkgreen.com/greenproducts.asp."

[40] EARTH Project, "http://www.ict-earth.eu/."

[41] TREND Project, "http://www.fp7-trend.eu." 


\section{Contents}

1 Introduction $\quad 4$

2 System Model and Methodology $\quad 5$

2.1 Basic formulation and metrics . . . . . . . . . . . . . . . 6

2.2 Node Switch-off Policy . . . . . . . . . . . . . . . . . . . . . . . 7

2.3 Generalization of the cost function . . . . . . . . . . . . . 9

3 Network Models $\quad 10$

3.1 The Erdös-Rényi model . . . . . . . . . . . . . . . . . . 11

3.2 Power-law model . . . . . . . . . . . . . . . . . . . . 12

3.3 The Watts-Strogatz model . . . . . . . . . . . . . . . . . . . . 12

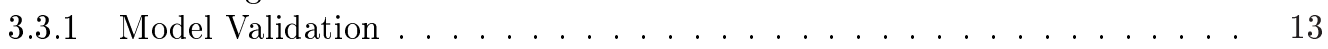

4 Performance Evaluation $\quad 17$

4.1 Impact of Sleep Modes . . . . . . . . . . . . . . . . . . . . . . . . 17

4.2 Impact of Technology Constraints . . . . . . . . . . . . . . . . . . . . . . . . . . . . . . . . .

4.3 Impact of Network Properties . . . . . . . . . . . . . . . . . . . . . . . 19

4.4 Impact of Power Consumption Model . . . . . . . . . . . . . . . . 20

5 Comparison of Switching Policies $\quad 21$

6 Discussion $\quad 22$

7 Related Work $\quad 23$

8 Conclusions $\quad 24$ 


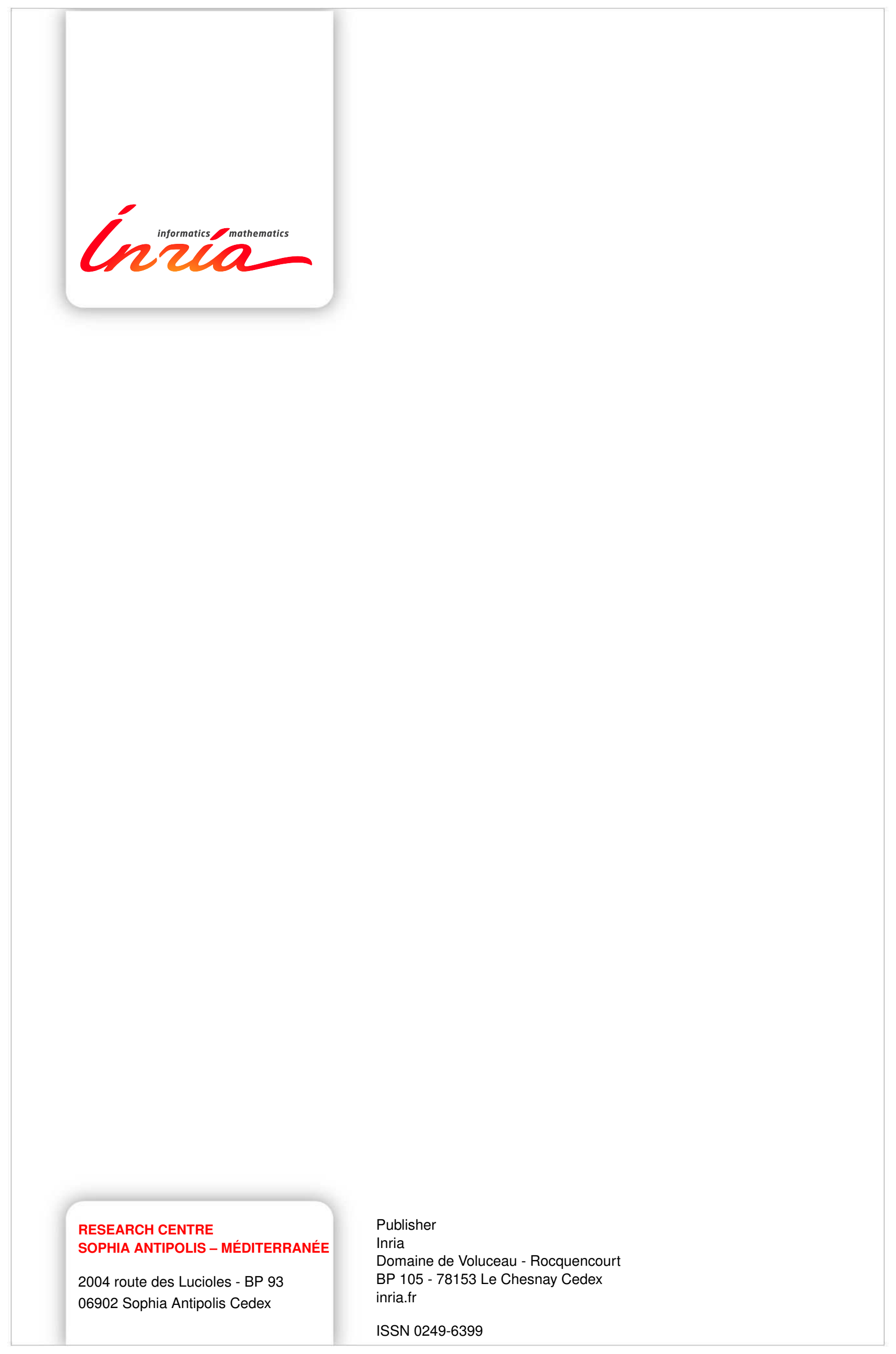

